\title{
THE HOPF BIFURCATION IN THE SHIMIZU-MORIOKA SYSTEM
}

\author{
JAUME LLIBRE \\ Departaments de Matematiques, Universitat Autònoma de Barcelona, \\ 08193 Bellaterra, Barcelona, Catalonia, Spain \\ jllibre@mat.uab.cat \\ Claudio Pessoa \\ Universidade Estadual Paulista, Departamento de Matemática \\ Campus São José do Rio Preto - IBILCE, \\ R. Cristóvão Colombo, 2265, 15.054000, São José do Rio Preto, SP, Brazil \\ pessoa@ibilce.unesp.br
}

\begin{abstract}
We study the local Hopf bifurcations of codimension one and two which occur in the Shimizu-Morioka system. This system is a simplified model proposed for studying the dynamics of the well known Lorenz system for large Rayleigh numbers. We present an analytic study and their bifurcation diagrams of these kinds of Hopf bifurcation, showing the qualitative changes in the dynamics of its solutions for different values of the parameters.
\end{abstract}

\section{INTRODUCTION}

In this paper we study the local Hopf bifurcations of codimension one and two and the kind of stability of the Hopf periodic orbits in the dynamics of the ShimizuMorioka system given by

$$
\dot{x}=y, \quad \dot{y}=x-\lambda y-x z, \quad \dot{z}=-\alpha z+x^{2},
$$

where $(x, y, z) \in \mathbb{R}^{3}$ are the state variables, and $\alpha$ and $\lambda$ are real parameters. System (1) is a simplified model proposed in [18] for studying the dynamics of the well known Lorenz system [9]. Later the system gained self-interest and several articles have appeared in the literature, dealing mainly with the chaotic behavior of the solutions and the emergence of strange attractor, see for instance $[6,17,18$, $20,21,22]$. It was shown in [17] among other properties that system (1) presents Lorenz-like strange attractors, for example taking $\alpha=0.45$ and $\lambda=0.75$ (see Figure 1 of [13]).

In this note we perform an analytic bifurcation analysis of dynamical aspects of the solutions of system (1), when the parameters vary, aiming to give a contribution to the understanding of its complex behavior. Our approach permits a geometric synthesis of the bifurcation analysis, based on the algebraic expression

2010 Mathematics Subject Classification. Primary 34C35, 58F09; Secondary 34D30.

Key words and phrases. Hopf bifurcation, limit cycles, bifurcation diagram. 
and geometric location of the codimension 2 Hopf point leading to the bifurcation of periodic orbits.

The study presented here is close to those realized in some papers, which was performed in [12] (see also [3]). But our approach is different, mainly in the computations of the Lyapunov coefficients which are necessary to study the Hopf bifurcations. In [12] the authors study the system

$$
\dot{x}=y-x, \quad \dot{y}=\beta x-x z, \quad \dot{z}=-\chi z+\eta x^{2} .
$$

This system and system (1) are equivalent if $\beta=\lambda=1$ and $\eta>0$, taking $\alpha=\chi$ and doing the change of variables $(x, y, z) \mapsto(\sqrt{\eta} x,-\sqrt{\eta} x+\sqrt{\eta} y, z)$ in system (1), but when $\beta \neq \lambda$ or $\eta \leq 0$ these systems are not equivalent.

Our main result is the following one.

Theorem 1. The following statements hold for system (1):

(a) For $\alpha=\frac{2-\lambda^{2}}{\lambda}$ and $\lambda \in(0, \sqrt{2})$ system (1) has two non-hyperbolic singular points $Q_{-}$and $Q_{+}$and, if $h(\lambda)=3 \lambda^{4}-5 \lambda^{2}-1 \neq 0$, a one codimension Hopf bifurcation take place at these points, permitting the existence of limit cycles near them. These cycles on the central manifolds of $Q_{-}$and $Q_{+}$are unstable if $h(\lambda)<0$ and stable if $h(\lambda)>0$.

(b) For $\alpha=\frac{2-\lambda^{2}}{\lambda}$ with $\lambda \in(0, \sqrt{2})$ and $h(\lambda)=0$ a two codimension Hopf bifurcation take place at the points $Q_{-}$and $Q_{+}$, with the creation of two limit cycles, one unstable and the other stable on the central manifolds of $Q_{-}$and $Q_{+}$.

The paper is organized as follows. In section 2 through a linear analysis of system (1) we present a study of the bifurcations which occurs with its singular points. In section 3 we describe a method to compute the focus quantities, related to the stability of the limit cycles which appear in the Hopf bifurcations. In section 4 we present a brief review of the theory used to study codimension one and two Hopf bifurcations. These methods are used in Section 5 to prove statements (a) and (b) of Theorem 1. For some extensions of the Hopf bifurcation see [1].

\section{AnAlysis OF THE SINGUlar POINTS}

The statement (a) and (b) of the next proposition are not new, in fact they are well know in the literature see for instance $[13,5]$.

Proposition 2. The following statements hold for system (1).

(a) For $\alpha<0$ the origin of system (1) is the unique hyperbolic singular point. It is a saddle with a one-dimensional stable manifold and two-dimensional unstable manifold;

(b) For $\alpha=0$ the $z$-axis of system (1) is filled of singular points. The origin becomes a non-hyperbolic singular point and a degenerate pitchfork bifurcation occurs on it. More precisely, for $\alpha>0$ sufficiently small, this line of singular points disappear, the origin becomes a hyperbolic saddle with a two-dimensional stable manifold and an one-dimensional unstable manifold and two new singular points $Q_{-}$and $Q_{+}$are created, they are symmetric with respect to the z-axis. These new equilibria are hyperbolic and asymptotically stable if $\alpha>\frac{2-\lambda^{2}}{\lambda}$ and $\lambda>0$. For either $\alpha=\frac{2-\lambda^{2}}{\lambda}$ and $\lambda \in(-\infty,-\sqrt{2})$ or $\alpha<\frac{2-\lambda^{2}}{\lambda}$ and $\lambda \in(-\infty,-\sqrt{2}) \cup(0, \sqrt{2}), Q_{-}$and $Q_{+}$ are unstable singular points. 
Proof. For $\alpha<0$ the origin $(0,0,0)$ is the unique singular point of system (1) and the eigenvalues of its linear part are

$$
\sigma_{0}=-\alpha, \quad \sigma_{ \pm}=\frac{-\lambda \pm \sqrt{\lambda^{2}+4}}{2},
$$

with eigenvectors given by $v_{0}=(0,0,1), v_{ \pm}=\left(1, \sigma_{ \pm}, 0\right)$, respectively. As the eigenvalues are all reals and $\alpha<0, \sigma_{+} \sigma_{-}<0$, by the Invariant Manifold Theorem and the Hartman Theorem (see for instance [7]), the origin is a hyperbolic saddle with an one-dimensional stable manifold tangent to the line generated by $v_{-}$and a two-dimensional unstable manifold tangent to the plane generated by $v_{0}$ and $v_{+}$ for all $\lambda$. Note that for $\alpha<0$ the solutions in the invariant $z$-axis go away from the origin.

If $\alpha=0$ the invariant $z$-axis is filled by singular points of system (1). Then the origin is a non-isolated degenerate singular point. Moreover, the eigenvalues of the linear part of system (1) at this point are 0 and $\sigma_{ \pm}$.

When the parameter $\alpha$ crosses the zero value the vector fields associated to system (1) cross this degenerate situation transversally. On the other words, for $\alpha>0$ the $z$-axis filled of singular points which exists for $\alpha=0$ disappears, and system (1) has only the singular points

$$
Q_{0}=(0,0,0), \quad Q_{ \pm}=( \pm \sqrt{\alpha}, 0,1) .
$$

The eigenvalues of the linear part of system (1) at $Q_{0}$ are given in (2) and we have $\sigma_{0}<0$ and $\sigma_{-}<0$ and $\sigma_{+}>0$. Therefore $Q_{0}$ is a hyperbolic saddle with a twodimensional stable manifold and an one-dimensional unstable manifold for all $\lambda$. Thus under the creation and subsequent elimination of the line of singular points when $\alpha$ crosses the zero value, the origin $Q_{0}$ of system (1) gains one dimension in the stable manifold and loses one dimension in the unstable one, as stated in statement (b) of the proposition.

Under the change of coordinates $(x, y, z) \mapsto(-x,-y, z)$, system (1) is invariant. Hence the kind of stability of the singular point $Q_{+}$follows from the kind of stability of $Q_{-}$. The characteristic polynomial of the linear part of system (1) at $Q_{-}$is

$$
p(\sigma)=-\sigma^{3}-(\alpha+\lambda) \sigma^{2}-\alpha \lambda \sigma-2 \alpha .
$$

The rest of proof follows from the next proposition.

Proposition 3. Consider $\alpha>0$. The singular point $Q_{-}$is asymptotically stable to system (1) if $\alpha>\frac{2-\lambda^{2}}{\lambda}$ and $\lambda>0$, and unstable if either $\alpha=\frac{2-\lambda^{2}}{\lambda}$ and $\lambda \in$ $(-\infty,-\sqrt{2})$ or $\alpha<\frac{2-\lambda^{2}}{\lambda}$ and $\lambda \in(-\infty,-\sqrt{2}) \cup(0, \sqrt{2})$.

Proof. The proof follows easily from the Routh-Hurwitz stability criterion (see [14] page 58).

The next proposition is a straightforward consequence of the relations between roots and coefficients of a polynomial in one variable.

Proposition 4. Consider $\alpha>0$. If $\alpha=\frac{2-\lambda^{2}}{\lambda}$ and $\lambda \in(0, \sqrt{2})$, then the linear part of system (1) at the singular point $Q_{-}$has one negative eigenvalue and two conugated pure imaginary eigenvalues.

Following [12], the symmetric bifurcation which occurs when the parameter $\alpha$ crosses the zero value is called degenerate pitchfork bifurcation, due to the line of equilibria which exists for $\alpha=0$, and it has already been observed in other systems which also present chaotic behavior (see for instance [16], page 4 and [12]). 


\section{Center Theorem and focus quantities}

In this section we summarize the method described in [4] (see also $[10,11]$ ) for studing the center problem on a center manifold for vector fields in $\mathbb{R}^{3}$. Let $X: U \rightarrow \mathbb{R}^{3}$ be a real analytic vector field, such that $D X(0)$ has two pure imaginary eigenvalues and one non-zero. By a linear change of variables and a possible rescaling of the time the system of differential equations $\dot{\mathbf{u}}=X(\mathbf{u})$ can be written as

$$
\begin{aligned}
\dot{u} & =-v+P(u, v, w)=\tilde{P}(u, v, w), \\
\dot{v} & =u+Q(u, v, w)=\tilde{Q}(u, v, w), \\
\dot{w} & =\beta w+R(u, v, w)=\tilde{R}(u, v, w),
\end{aligned}
$$

where $\beta$ is a real non-zero number. We denote again by $X$ this new vector field.

A non-constant $C^{1}$ function $H$ from a neighborhood of the origin of $\mathbb{R}^{3}$ into $\mathbb{R}$ is a local first integral of system (3) if it is constant on the orbits of (3), i.e. $H$ satisfies

$$
X H=\tilde{P} \frac{\partial H}{\partial u}+\tilde{Q} \frac{\partial H}{\partial v}+\tilde{R} \frac{\partial H}{\partial w} \equiv 0,
$$

in a neighborhood of the origin. A non-constant formal power series $H$ in $u, v$ and $w$ is a formal first integral for system (3) if when $\tilde{P}, \tilde{Q}$, and $\tilde{R}$ are expanded in power series at the origin, every coefficient in the formal power series in (4) is zero. If $w$ and $\dot{w}$ do not appear in system (3) the system is in $\mathbb{R}^{2}$, the singular point at the origin is either a focus (every trajectory near the origin spirals towards the origin, or every trajectory does so in reverse time) or a center (a punctured neighborhood is composed entirely of periodic orbits). The problem of distinguishing between these two cases is the center problem. It was solved by Poincaré and Lyapunov in terms of the non-existence or existence of a local first integral. A proof is given in [15].

From Theorem 5.1 page 152 of [7] we know that system (3) admits a local center manifold $W_{l o c}^{c}$ at the origin. The following theorem provides one the main tools for detecting a center on a center manifold. See [4] for a proof.

Theorem 5. The following statements are equivalent.

(a) The origin is a center for $\left.X\right|_{W_{l o c}^{c}}$.

(b) There is a local analytic first integral at the origin for system (3) of the form $H(u, v, w)=u^{2}+v^{2}+\cdots$ (here the dots mean higher order terms).

(c) There is a formal first integral at the origin for system (3) of the form $H(u, v, w)=u^{2}+v^{2}+\cdots$.

The Lyapunov Center Theorem correspond to the equivalence of statements (a) and (b); for a proof see also [2]. From this theorem we can restrict our attention to investigate the conditions for the existence or non-existence of a first integral of the form $H(u, v, w)=u^{2}+v^{2}+\cdots$, which is equivalent to determine necessary and sufficient conditions for the existence of a center or a focus on the local center manifold, respectively.

In what follows we consider that $\mathrm{P}, \mathrm{Q}$ and $\mathrm{R}$ in (3) are polynomials. We start by introducing the complex variable $x=u+i v$. Therefore the first two equations in (3) are equivalent to the unique equation $\dot{x}=i x+\cdots$. Adding to this equation its complex conjugate, changing $\bar{x}$ (where as usual $\bar{x}$ denote the conjugate of $x$ ) by 
$y$, thinking in $y$ as an independent complex variable, and substituting $w$ by $z$, we obtain the following complexification of system (3)

$$
\begin{aligned}
& \dot{x}=i x+\sum_{p+q+r=2}^{n} a_{p q r} x^{p} y^{q} z^{r}, \\
& \dot{y}=-i y+\sum_{p+q+r=2}^{n} b_{p q r} x^{p} y^{q} z^{r}, \\
& \dot{z}=\beta z+\sum_{p+q+r=2}^{n} c_{p q r} x^{p} y^{q} z^{r},
\end{aligned}
$$

where $b_{q p r}=\bar{a}_{p q r}$ and the $c_{p q r}$ are such that $\sum_{p+q+r=2}^{n} c_{p q r} x^{p} \bar{x}^{q} w^{r}$ is real for all $x \in \mathbb{C}$ and $w \in \mathbb{R}$. Again we denote by $X$ the new vector field associated to system (5) on $\mathbb{C}^{3}$. Now the existence of a first integral $H(u, v, w)=u^{2}+v^{2}+\cdots$ for a system (3) is equivalent to the existence of a first integral of the form

$$
H(x, y, z)=x y+\sum_{j+k+l=3} v_{j k l} x^{j} y^{k} z^{l}
$$

for system (5).

By computing the coefficients of $X H$ and equating them to zero we investigate the existence of a first integral $H$ for a system (5). When $H$ has the form (6) we can calculated explicitly the coefficient $g_{k_{1} k_{2} k_{3}}$ of $x^{k 1} y^{k 2} z^{k 3}$ in $X H$ (see [4]). But when $\left(k_{1}, k_{2}, k_{3}\right)=(k, k, 0)$ for a positive integer $k$, we can solved in a unique way for $v_{k_{1} k_{2} k_{3}}$ the equation $g_{k_{1} k_{2} k_{3}}=0$ in terms of the known quantities $v_{\alpha \beta \gamma}$ such that $\alpha+\beta+\lambda<k_{1}+k_{2}+k_{3}$. Hence if $g_{k k 0}=0$ for all $k \in \mathbb{N}$ a formal first integral $H$ exists. When the coefficient $g_{k k 0}$ is non-zero an obstruction to the existence of the formal series $H$ occurs. Such a coefficient is called the kth focus quantity.

The focus quantities $g_{110}=0$ and $g_{220}$ are determined in a unique way, but the others depend on the choices made for $v_{k k 0}, k \in \mathbb{N}, k \geq 2$. Once such computations are made, $H$ is determined and satisfies

$$
X H(x, y, z)=g_{220}(x y)^{2}+g_{330}(x y)^{3}+\cdots .
$$

It follows that if for one choice of the $v_{k k 0}$ at least one focus quantity is non-zero, the same is true for every other choice of the $v_{k k 0}$. A sufficient and necessary condition for the existence of a center on the center manifold is to vanish all focus quantities, otherwise we have a focus (see [4]).

In rest of this work we denote the kth focus quantity $g_{k k 0}$ by $\nu_{k}$.

\section{Hopf Bifurcation Method}

Let $(\theta, \rho)$ be polar coordinates on the local center manifold $W_{\text {loc }}^{c}$, such that $\rho=0$ corresponds to the origin in cartesian coordinates. Consider system (3) restricted to its local center manifold and let $\Pi(\rho)$ the respective Poincaré first return map on a sufficiently short segment of the axis $\theta=0$ starting at $\rho=0$. By the $k$ th Lyapunov coefficient we mean the coefficient $l_{k}$ in the expansion of displacement map $\Pi(\rho)-\rho$, i.e.

$$
\Pi(\rho)-\rho=l_{1} \rho+l_{2} \rho^{2}+\cdots .
$$

It follows by the proof of Theorem 6.2.3 of the page 261 of [15] that

$$
l_{1}=c_{1} \nu_{1} \text { and }\left.l_{k}\right|_{l_{1}=\cdots=l_{k-1}=0}=\left.c_{k} \nu_{k}\right|_{\nu_{1}=\cdots=\nu_{k-1}=0},
$$


where $c_{1}, \ldots, c_{k}$ are positive constants.

A method to compute the Lyapunov coefficients can be found in the pages 177181 of [7] or in [8, 12].

A singular point $\left(x_{0}, \mu_{0}\right)$ of a $\mu$-parameter family of vector fields $X(x, \mu)$ in $\mathbb{R}^{3}$ is called a Hopf point if the Jacobian matrix $D X\left(x_{0}, \mu_{0}\right)$ has a real eigenvalue $\lambda_{1} \neq 0$ and a pair of purely imaginary eigenvalues $\lambda_{2,3}= \pm i \omega_{0}$. There is a twodimensional center manifold at a Hopf point and it is invariant by the flow of the system $\dot{x}=X(x, \mu)$, see page 152 of [7]. If varying the parameters the complex eigenvalues cross the imaginary axis with non-zero derivative, the Hopf point is called transversal, i.e. if $\mu$ is one-dimensional parameter then $\frac{d \xi}{d \mu}\left(\mu_{0}\right) \neq 0$ (where $\xi(\mu) \pm i \omega(\mu)$ are the conjugated complex eigenvalues of the linear part of $X(x, \mu)$ at singular point $x_{\mu}$ when $\left|\mu-\mu_{0}\right|$ is enough small). At a neighborhood of transversal Hopf point with $l_{1}\left(\mu_{0}\right) \neq 0$ the system $\dot{x}=X(x, \mu)$ restricted to a center manifold, is orbitally topologically equivalent to the following complex normal form

$$
\dot{w}=(\xi+i \omega) w+\sigma w|w|^{2},
$$

where $w \in \mathbb{C}, \sigma=\operatorname{sign} l_{1}\left(\mu_{0}\right)= \pm 1, l_{1}\left(\mu_{0}\right)$ the first Lyapunov coefficient at the Hopf point, and $\xi, \omega$ are real functions having derivatives of arbitrary higher order, which are continuations of 0 and $\omega_{0}$, see page 98 of [7]. There is one family of stable (unstable) periodic orbits if $l_{1}<0\left(l_{1}>0\right)$ on the space of phases variables and parameters shrinking to a singular Hopf point.

A Hopf point of codimension 2 is a Hopf point where $l_{1}\left(\mu_{0}\right)=0$ and $l_{2}\left(\mu_{0}\right) \neq 0$. It is called transversal if the manifolds $\xi(\mu)=0(\xi(\mu)$ is the real part of the conjugated complex eigenvalues) and $l_{1}(\mu)$ have transversal intersections, i.e. the map $\mu \mapsto\left(\xi(\mu), l_{1}(\mu)\right)$ is regular at $\mu_{0}$. The system $\dot{x}=X(x, \mu)$ restricted to a center manifold at a neighborhood of a transversal Hopf point of codimension 2 is orbitally topologically equivalent to

$$
\dot{w}=\left(\xi+i \omega_{0}\right) w+\tau w|w|^{2}+\sigma w|w|^{4},
$$

where $\xi$ and $\tau$ are the unfolding parameters and $\sigma=\operatorname{sign} l_{2}\left(\mu_{0}\right)= \pm 1$, see page 311 of [7]. The bifurcation diagram of system (8) on the space of parameters $(\xi, \tau)$ for $\sigma=1$ is showed in Figure 1. Where the lines $H_{1}^{ \pm}=\{ \pm \tau>0\}$ correspond to the Hopf bifurcation of codimension one with negative and with positive Lyapunov coefficient, respectively. Along these lines the singular point has eigenvalues $\lambda_{1,2}=$ $\pm \omega_{0} i$. Moreover the singular point is stable for $\xi<0$ and unstable for $\xi>0$. The first Lyapunov coefficient is $l_{1}(\xi, \tau)=\tau$. Therefore the point of the Hopf bifurcation of codimension two $H_{2}$ occurs when $\xi=\tau=0$ and separates the two branches, $H_{1}^{-}$ and $H_{1}^{+}$of $\tau$-axis. An unstable limit cycle bifurcates from the singular point if we cross $H_{1}^{+}$from right to left, while a stable limit cycle appears if we cross $H_{1}^{-}$in the opposite direction. These limit cycles collide and disappear on the curve

$$
T=\left\{(\xi, \tau): 4 \xi-\tau^{2}=0\right\},
$$

corresponding to a nondegenerate fold bifurcation of the cycles. Along this curve the system has a semistable limit cycle of multiplicity one, see page 311 of [7].

The bifurcation diagrams for $\sigma=-1$ can be found in [7], page 313, and in [19] .

From (7) the Hopf method described above can be applied changing the Lyapunov coefficients by the focus quantities. Thus in rest of this paper we shall use the focus quantities in place of the Lyapunov coefficients to study Hopf bifurcations of codimension one and two. 


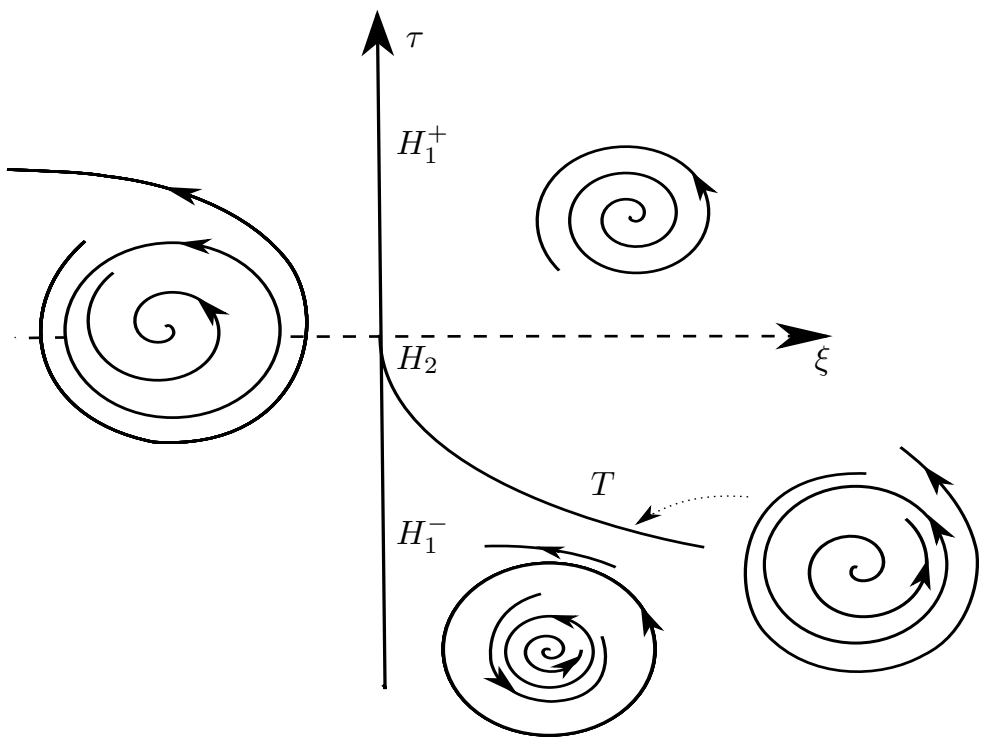

FiguRE 1. Diagram at the point $H_{2}$ of a two codimension Hopf bifurcation.

\section{Hopf Bifuraction in the Shimizu-Morioka system}

In this section we study the stability of the singular point $Q_{-}$of system (1) under the conditions $\alpha=\frac{2-\lambda^{2}}{\lambda}$ and $\lambda \in(0, \sqrt{2})$ given in Preposition 4, i.e. on the Hopf axis correspondent to the $\tau$-axis of Figure 1. We prove the following theorem.

Theorem 6. Consider the two-parameter family of differential equations (1). The first focus quantity at the point $Q_{-}$for parameter values satisfying $\alpha=\frac{2-\lambda^{2}}{\lambda}$ and $\lambda \in(0, \sqrt{2})$ is given by

$$
\nu_{1}(\lambda)=\frac{\lambda \sqrt{2-\lambda^{2}}\left(3 \lambda^{4}-5 \lambda^{2}-1\right)}{4\left(\lambda^{4}-2 \lambda^{2}-4\right)\left(\lambda^{4}-2 \lambda^{2}-1\right)} .
$$

For $\lambda \in(0, \sqrt{2})$ such that $h(\lambda)=3 \lambda^{4}-5 \lambda^{2}-1$ is different from zero, system (1) has a transversal Hopf point at $Q_{-}$for $\alpha=\frac{2-\lambda^{2}}{\lambda}$.

Now for the parameter values $\lambda_{c}=\sqrt{\frac{5+\sqrt{37}}{6}}$ and $\alpha=\frac{7-\sqrt{37}}{\sqrt{6(5+37)}}$ system (1) has a transversal Hopf point of codimension 2 at $Q_{-}$which is unstable because $\nu_{2}>0$.

Proof. For simplify the computations, we introduce the new parameters $(\beta, \varepsilon)$ by

$$
\begin{aligned}
& \lambda=\frac{-\varepsilon\left(\beta^{2}+\varepsilon^{2}+2\right)+\sqrt{\left(-\beta^{2}-\varepsilon^{2}+2\right)\left(\beta^{4}+\left(\beta^{2}+2\right) \varepsilon^{2}\right)}}{\beta^{2}+\varepsilon^{2}}, \\
& \alpha=-\varepsilon-\frac{\sqrt{\left(-\beta^{2}-\varepsilon^{2}+2\right)\left(\beta^{4}+\left(\beta^{2}+2\right) \varepsilon^{2}\right)}}{\beta^{2}+\varepsilon^{2}-2} .
\end{aligned}
$$


The Jacobian determinant of this change of parameters in the point $(0, \beta)$ is

$$
-\frac{2 \beta^{5}\left(\beta^{4}-2 \beta^{2}-4\right)}{\left(-\beta^{4}\left(\beta^{2}-2\right)\right)^{3 / 2}} .
$$

Thus the change of parameters is well defined for $(\varepsilon, \beta) \in(-\delta, \delta) \times(0, \sqrt{2})$, with $\delta$ enough small. In this new parameters the linear part of system (1) at the singular point $Q_{-}$has a real eigenvalue and two conjugated complex given by $\varepsilon \pm i \beta$. Furthermore the conditions $\alpha=\frac{2-\lambda^{2}}{\lambda}$ and $\lambda \in(0, \sqrt{2})$ correspond to $\varepsilon=0$ and $\beta \in(0, \sqrt{2})$. Hence, in this case, we have

$$
\alpha=\frac{\beta^{2}}{\sqrt{2-\beta^{2}}} \quad \text { and } \quad \lambda=\sqrt{2-\beta^{2}}
$$

in system (1). Now, doing the change of coordinates $(x, y, z) \mapsto(\tilde{x}-\sqrt{\alpha}, \tilde{y}, \tilde{z}+1)$, the singular point $Q_{-}$is translated to the origin $(0,0,0)$. Now we shall write the linear part of system in the coordinates $(\tilde{x}, \tilde{y}, \tilde{z})$ at the origin in its real Jordan normal form. For this we introduce the variables $(u, v, w)$ by

$$
\left(\begin{array}{c}
\tilde{x} \\
\tilde{y} \\
\tilde{z}
\end{array}\right)=\left(\begin{array}{ccc}
-\frac{\beta}{2 \sqrt[4]{2-\beta^{2}}} & \frac{1}{2} \sqrt[4]{2-\beta^{2}} & 2\left(2-\beta^{2}\right)^{5 / 4} \\
\frac{1}{2} \beta \sqrt[4]{2-\beta^{2}} & \frac{\beta^{2}}{2 \sqrt[4]{2-\beta^{2}}} & -4\left(2-\beta^{2}\right)^{3 / 4} \\
1 & 0 & 4 \beta \sqrt{2-\beta^{2}}
\end{array}\right)\left(\begin{array}{c}
u \\
v \\
w
\end{array}\right)
$$

and so system (1) becomes

$$
\begin{aligned}
\dot{u}= & -v-\frac{\beta \sqrt{2-\beta^{2}}\left(6+\beta^{2}\right) u^{2}}{-16-8 \beta^{2}+4 \beta^{4}}-\frac{\left(-8+2 \beta^{2}+\beta^{4}\right) v u}{-8-4 \beta^{2}+2 \beta^{4}} \\
& \left.+\frac{2\left(-2+\beta^{2}\right)\left(-8+4 \beta^{2}+\beta^{4}\right) w u}{-4-2 \beta^{2}+\beta^{4}}+\frac{\sqrt{2-\beta^{2}}\left(-4+\beta^{4}\right) v^{2}}{\beta\left(-16-8 \beta^{2}+4 \beta^{4}\right.}\right) \\
& -\frac{2 \sqrt{2-\beta^{2}}\left(8-8 \beta^{2}+\beta^{6}\right) w v}{\beta\left(-4-2 \beta^{2}+\beta^{4}\right)}+\frac{4\left(2-\beta^{2}\right)^{5 / 2}\left(-4+4 \beta^{2}+\beta^{4}\right) w^{2}}{\beta\left(-4-2 \beta^{2}+\beta^{4}\right)}, \\
\dot{v}= & u+\frac{\left(8+\beta^{4}\right) u^{2}}{16+8 \beta^{2}-4 \beta^{4}}+\frac{\sqrt{2-\beta^{2}}}{\beta\left(-8-4 \beta^{2}+2 \beta^{4}\right)} \\
& -\frac{2 \sqrt{2-\beta^{2}}\left(-8+8 \beta^{2}-2 \beta^{4}+\beta^{6}\right) w u}{\beta\left(-4-2 \beta^{2}+\beta^{4}\right)}+\frac{\beta^{2}\left(-2+\beta^{2}\right) v^{2}}{4\left(-4-2 \beta^{2}+\beta^{4}\right)} \\
& -\frac{2\left(-8+8 \beta^{2}-4 \beta^{4}+\beta^{6}\right) v w}{-4-2 \beta^{2}+\beta^{4}}+\frac{4\left(-2+\beta^{2}\right)^{2}\left(8-2 \beta^{2}+\beta^{4}\right) w^{2}}{-4-2 \beta^{2}+\beta^{4}}, \\
\dot{w}= & -\frac{2 w}{\beta \sqrt{2-\beta^{2}}+\frac{\left(-4+3 \beta^{2}\right) u^{2}}{64-32 \beta^{4}+8 \beta^{6}}+\frac{\left(-1+\beta^{2}\right) v u}{2 \beta \sqrt{2-\beta^{2}}\left(-4-2 \beta^{2}+\beta^{4}\right)}} \\
& +\frac{\left(-4+8 \beta^{2}-3 \beta^{4}\right) w u}{\beta \sqrt{2-\beta^{2}}\left(-4-2 \beta^{2}+\beta^{4}\right)}-\frac{6\left(-2+\beta^{2}\right)^{2} w^{2}}{-4-2 \beta^{2}+\beta^{4}} \\
& +\frac{v^{2}}{32+16 \beta^{2}-8 \beta^{4}+\frac{2\left(-2+\beta^{2}\right) v w}{-4-2 \beta^{2}+\beta^{4}} .}
\end{aligned}
$$


Note that the above system is in the form (3). Now we apply the method described in section 3 .

Firstly we introduce the change of variables $(u, v, w) \mapsto(x, y, z)=(u+i v, u-$ $i v, w)$ with inverse given by $(x, y, z) \mapsto(u, v, w)=\left(\frac{1}{2}(x+y),-\frac{i}{2}(x-y), z\right)$. Hence we obtain system (1) in the complex form (5). Aigain denote by $X$ the vector field associated to this last system in complex form and let $H$ be given by (6). We have that

$$
X H(x, y, z)=\sum_{m \geq 2} H_{m}(x, y, z),
$$

where, $H_{m}$ are homogeneous polynomials of degree $m$ in the variables $(x, y, z)$. It is easy to see that $H_{2} \equiv 0$. Denoting the coefficients of $H_{m}$ by $g_{j k l}$ with $j+k+l=m$, we can solve easily the equations $g_{j k l}=0$ with $j+k+l=3$ in terms of the coefficients $\nu_{\alpha \beta \gamma}$ of $H$ such that $\alpha+\beta+\gamma \leq 3$. For instance the equation $g_{003}=0$ is given by

$$
-\frac{6 \nu_{003}}{\beta \sqrt{2-\beta^{2}}}=0
$$

which solution in terms of the coefficients of $H$ is $\nu_{003}=0$. Analogously, we can solve the equations $g_{j k l}=0$ with $j+k+l=4$ in terms of the coefficients $\nu_{\alpha \beta \gamma}$ of $H$ with $\alpha+\beta+\gamma \leq 4$, except the equation $g_{220}=0$, because this equation does not depend on the coefficients of $H$, only on the coefficients of $X$. Hence we have that the first focus quantity is $g_{220}=\nu_{1}$ given by

$$
\nu_{1}=\frac{\sqrt{2-\beta^{2}}\left(3 \beta^{4}-7 \beta^{2}+1\right) \beta}{4\left(\beta^{4}-2 \beta^{2}-4\right)\left(\beta^{4}-2 \beta^{2}-1\right)} .
$$

Following the above ideas we obtain the second focus quantity $g_{330}=\nu_{2}$, i.e.

$$
\begin{aligned}
\nu_{2}= & \left(\sqrt { 2 - \beta ^ { 2 } } \left(-162 \beta^{22}+2268 \beta^{20}-14289 \beta^{18}+47071 \beta^{16}-80155 \beta^{14}\right.\right. \\
& +63495 \beta^{12}-20967 \beta^{10}+16999 \beta^{8}-23136 \beta^{6}+9300 \beta^{4}+9760 \beta^{2} \\
& -80)) /\left(96 \beta\left(\beta^{4}-2 \beta^{2}-4\right)^{3}\left(\beta^{4}-2 \beta^{2}-1\right)^{2}\left(9 \beta^{2}\left(\beta^{2}-2\right)-4\right)\right) .
\end{aligned}
$$

Note that the polynomial $\beta^{4}-2 \beta^{2}-4$ has only two real roots, $\pm \sqrt{1+\sqrt{5}}$, and so it has negative sign in $(-\sqrt{1+\sqrt{5}}, \sqrt{1+\sqrt{5}})$. Now the polynomial $\beta^{4}-$ $2 \beta^{2}-1$, also has only two real roots, $\pm \sqrt{1+\sqrt{2}}$, and so it has negative sign in $(-\sqrt{1+\sqrt{2}}, \sqrt{1+\sqrt{2}})$. Therefore the sign of the first focus quantity is determined by the sign of $h(\beta)=3 \beta^{4}-7 \beta^{2}+1$, since we are considering $\beta \in(0, \sqrt{2})$ and so the denominator of $(10)$ is positive. Observe that, for $\beta \in(0, \sqrt{2})$, the first focus quantity vanishes on $\beta_{c}=\sqrt{\frac{1}{6}(7-\sqrt{37})}$ and the second is deferent from zero, i.e. $\nu_{1}\left(\beta_{c}\right)=0$ and $\nu_{2}\left(\beta_{c}\right)=\frac{\sqrt{274249 \sqrt{37}-591726}}{15568}$. Moreover $\nu_{1}>0$ for $\beta \in\left(0, \beta_{c}\right)$ and $\nu_{1}<0$ for $\beta \in\left(\beta_{c}, \sqrt{2}\right)$.

Clearly, in the plane of parameters $(\varepsilon, \beta)$, we have a transversal Hopf point in $Q_{-}$for $\varepsilon=0$ and $\beta \in(0, \sqrt{2}) \backslash\left\{\beta_{c}\right\}$. Now as the map $(\varepsilon, \beta) \mapsto\left(\varepsilon, \nu_{1}(\beta)\right)$ is regular in $\left(0, \beta_{c}\right)$, since

$$
\frac{d \nu_{1}}{d \beta}\left(\beta_{c}\right)=-\frac{\sqrt{37(14066 \sqrt{37}-84205)}}{1946},
$$


it follows that we have a transversal Hopf point of codimension 2 in $Q_{-}$for $\varepsilon=0$ and $\beta=\beta_{c}$.

In the parameter $\lambda$, by (9), the function $h$ becomes $h(\lambda)=3 \lambda^{4}-5 \lambda^{2}-1 \neq 0$,

$$
\nu_{1}(\lambda)=\frac{\lambda \sqrt{2-\lambda^{2}}\left(3 \lambda^{4}-5 \lambda^{2}-1\right)}{4\left(\lambda^{4}-2 \lambda^{2}-4\right)\left(\lambda^{4}-2 \lambda^{2}-1\right)}
$$

and $\nu_{1}(\lambda)$ is zero in the $(0, \sqrt{2})$ only for the value $\lambda_{c}=\sqrt{\frac{5+\sqrt{37}}{6}}$. Moreover in this point, by $(9), \alpha=\frac{7-\sqrt{37}}{\sqrt{6(5+37)}}$.

The same results stated in Theorem 1 are valid also for the point $Q_{+}$, due to the symmetry of the system under the change $(x, y, z) \mapsto(-x,-y, z)$. The statements $(a)$ and $(b)$ of Theorem 1 follow from the above results.

The bifurcation diagram on the space of parameters $(\lambda, \alpha)$ of system $(1)$ on the neighborhood of the two codimension Hopf point $H_{2}=\left(\sqrt{\frac{5+\sqrt{37}}{6}}, \frac{7-\sqrt{37}}{\sqrt{6(5+37)}}\right)$ is described in Figure 2, where by section 4 the curves $H_{1}^{ \pm}$and $T$ correspond respectively with the curves of Figure 1. Note that

$$
H_{1}^{-} \cup H_{2} \cup H_{1}^{+}=\left\{(\lambda, \alpha): \alpha=\frac{2-\lambda^{2}}{\lambda}, \lambda \in(0, \sqrt{2})\right\} .
$$

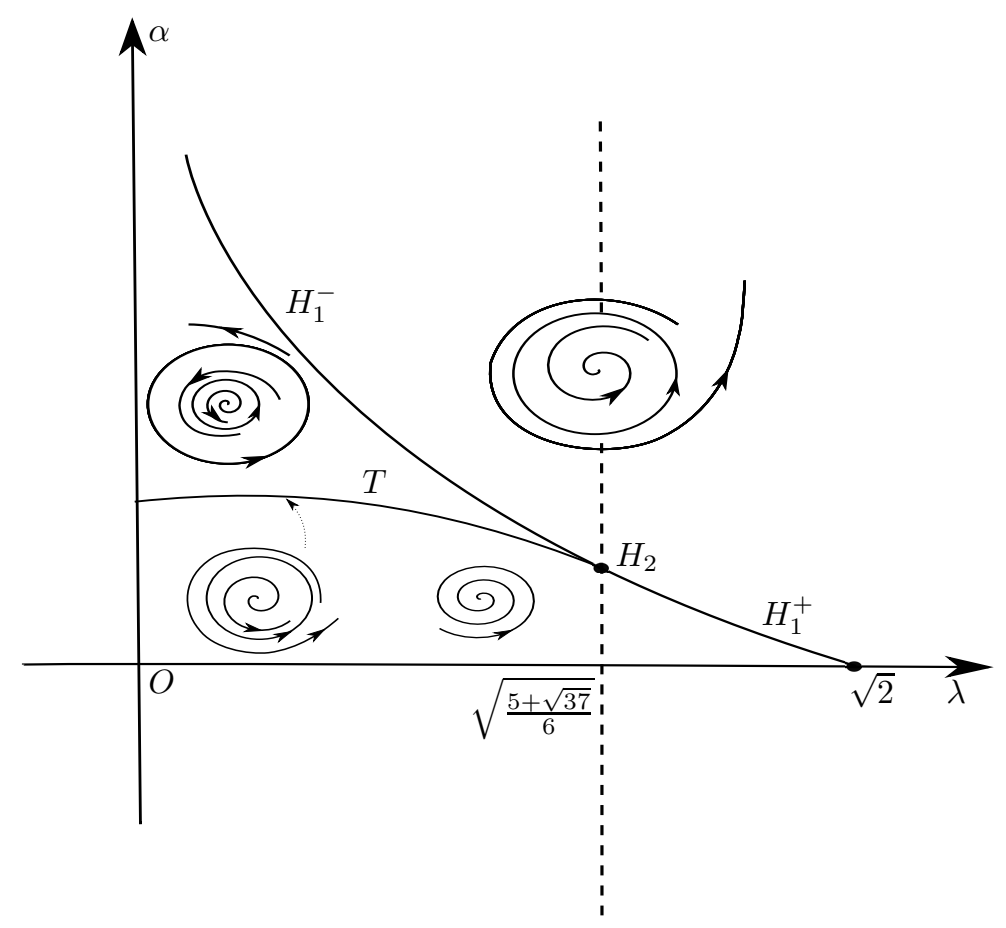

Figure 2. Diagram at the point $H_{2}$ of the two codimension Hopf bifurcation of system (1). 


\section{ACKNOWLEDGMENTS}

The first author is partially supported by the grants MINECO/FEDER MTM 2008-03437, AGAUR 2014SGR 568, ICREA Academia and FP7 PEOPLE-2012IRSES-316338 and 318999. The second author is partially supported by Program CAPES/DGU Process 8333/13-0 and by FAPESP Project 2011/13152-8.

\section{REFERENCES}

[1] Aguirre, L., Arredondo, J. H., López, R. and Seibert, P.: On certain generalizations of the Hopf bifurcation. Ann. Mat. Pura Appl. 186, 509-524 (2007).

[2] Bibikov, Y. : Local Theory of Nonlinear Analytic Ordinary Differential Equations. Lecture Notes in Mathematics, Vol. 702.

[3] Dias, F. S., Mello, L. F. and Zhang, J-G.: Nonlinear analysis in a Lorenz-like system. Nonlinear Anal. Real World Appl. 11 (5), 3491-3500 (2010).

[4] Edneral, V., Mahdi, A., Romanovski, V. G. and Shafer, D.S.: The center problem on a center mani- fold. Nonlin. Anal.: Th. Meth. Appl. 75, 26142622 (2012).

[5] Islam, N., Mazumdar, H. P. and Das, A.: On the stability and control of the Schimizu-Morioka system of dynamical equations. Differ. Geom. Dyn. Syst. 11 , 135-143 (2009).

[6] Kokubu, H. and Roussarie, R.: Existence of a singularly degenerate heteroclinic cycle in the Lorenz system and its dynamical consequences: Part I. J. Dyn. Diff. Eqn. 16(2), 513-557 (2004).

[7] Kuznetsov, Y.A., Elements of applied bifurcation theory. New York: SpringerVerlag, 2004.

[8] Kuznetsov, Y.A.: Numerical normalization techniques for all codim 2 bifurcations of equilibria in ODE's. SIAM J. Numer. Anal. 36 , 1104-1124 (1999).

[9] Lorenz, E.N.: Deterministic nonperiodic flow. J. Atmos. Sci. 20, 130-141 (1963).

[10] Mahdi, A.: Center problem for third-order ODEs. Internat. J. Bifur. Chaos Appl. Sci. Engrg. 23(5), 11 pp. (2013).

[11] Mahdi, A., Romanovski, V.G. and Shafer, D.S.: Stability and periodic oscillations in the Moon-Rand systems. Nonlinear Anal. Real World Appl. 14 (1), 294-313 (2013).

[12] Mello, L. F., Messias, M. and Braga, D.C.: Bifurcation analysis of a new Lorenz-like chaotic system. Chaos, Solitons and Fractals 37, 1244-1255 (2008).

[13] Messias, M., Gouveia, M. R. A. and Pessoa, C.: Dynamics at infinity and other golbal dynamical aspects of Shimizu-Morioka equations. Nonlinear Dyn. 69, 577-587 (2012).

[14] Pontryagin, L.S., Ordinary differential equations. Reading: Addison-Wesley Publishing Company Inc., 1962.

[15] Romanovski, V.G. and Shafer, D.S.: The Center and Cyclicity Problems: A Computational Algebra Approach. Birkhäuser, Boston, 2009.

[16] Rubinger, R.M., Nascimento, A.W.M, Mello L.F., Rubinger C.P.L, Manzanares Filho N. and lbuquerque H.A.: Inductorless Chua's circuit: experimental time series analysis. Mathematical Problems in Engineering (2007).

[17] Shilnikov, A. L.: On bifurcations of the Lorenz attractor in the ShimizuMorioka model. Physica D: Nonlinear Phenomena 62, 338-346 (1993). 
[18] Shimizu, T. and Morioka, N.: On the bifurcation of a symmetric limit cycle to an asymmetric one in a simple model. Physics Letters A 76(3,4), 201-204 (1980).

[19] Takens, F.: Unfoldings of certain singularities of vectorfields: Generalized Hopf bifurcations. J. Differ. Eq. 14, 476-93 (1973).

[20] Tigan, G. and Turaev, D.: Analytical search for homoclinic bifurcations in the Shimizu-Morioka model. Physica D: Nonlinear Phenomena 240(12), 985-989 (2011).

[21] Vladimirov, A. G. and Volkov, D.Y.: Low-intensity chaotic operations of a laser with a saturable absorber. Optics Communications 100, 351-360 (1993).

[22] Yu, S., Tang, W. K. S., Lü, J. and Chen, G.: Generation of $n \times m$-wing Lorenz-like attractors from a modified Shimizu-Morioka model. IEEE Trans. Circ. Systems 55 (11), 1168-1172, (2008). 